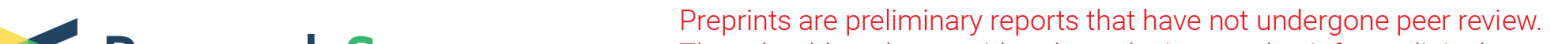 $\begin{array}{ll}\text { Research Square } & \text { They should not be considered conclusive, used to inform clinical practice, } \\ \text { or referenced by the media as validated information. }\end{array}$
}

\section{All-in-One Dual CRISPR-Cas12a (AIOD-CRISPR) Assay: A Case for Rapid, Ultrasensitive and Visual Detection of Novel Coronavirus SARS-CoV-2 and HIV virus at the Point of Care}

\section{Xiong Ding}

Department of Biomedical Engineering, University of Connecticut Health Center, 263 Farmington Ave., Farmington, CT, 06030, United States

Kun Yin

Department of Biomedical Engineering, University of Connecticut Health Center, 263 Farmington Ave., Farmington, CT, 06030, United States

\section{Ziyue Li}

Department of Biomedical Engineering, University of Connecticut Health Center, 263 Farmington Ave., Farmington, CT, 06030, United States

\section{Rajesh V. Lalla}

Section of Oral Medicine, University of Connecticut Health Center, Farmington, Connecticut 06030, United States

\section{Enrique Ballesteros}

Department of Pathology and Laboratory Medicine, University of Connecticut Health Center, Farmington, Connecticut 06030, United States

\section{Maroun M. Sfeir}

Department of Pathology and Laboratory Medicine, University of Connecticut Health Center, Farmington, Connecticut 06030, United States

Changchun Liu ( $\nabla$ chaliu@uchc.edu )

Department of Biomedical Engineering, University of Connecticut Health Center, 263 Farmington Ave., Farmington, CT, 06030, United States

\section{Research Article}

Keywords: All-In-One Dual CRISPR-Cas12a assay, virus detection, SARS-CoV- 2, HIV-1 virus

Posted Date: April 28th, 2020

DOI: https://doi.org/10.21203/rs.3.rs-25826/v1 
License: (c) (i) This work is licensed under a Creative Commons Attribution 4.0 International License. Read Full License

Version of Record: A version of this preprint was published on September 18th, 2020. See the published version at https://doi.org/10.1038/s41467-020-18575-6. 


\section{Abstract}

The recent outbreak of novel Coronavirus (SARS-CoV-2), the causative agent of COVID-19 disease, has spread rapidly all over the world. Human immunodeficiency virus (HIV) is another deadly virus and causes acquired immunodeficiency syndrome (AIDS). Rapid and early detection of these viruses will facilitate early intervention and prevent disease spread. Here, we present an All-In-One Dual CRISPRCas12a (termed "AIOD-CRISPR") assay method for simple, rapid, ultrasensitive, specific, one-pot, and visual detection of coronavirus SARS-CoV- 2 and HIV-1 virus. In our AIOD-CRISPR assay, a pair of crRNAs was introduced to initiate dual CRISPR-Cas12a-based detection and improve both detection sensitivity and fluorescence signals. The AIOD-CRISPR assay method was utilized to detect nucleic acids (DNA and RNA) of the SARS-CoV-2 and HIV-1 with a sensitivity of few copies. We validated our AIOD-CRISPR method by using COVID-19 swab samples and obtained consistent results with that of RT-PCR method. More importantly, we successfully demonstrated to use a low- cost hand warmer ( \$ 0.3) as an incubator of our AIOD-CRISPR assay and detect COVID-19 patient samples within 20 minutes, enabling an instrument-free, visual detection of COVID-19 at the point of care. Thus, our method has significant potential for developing next-generation point-of-care molecular diagnostics.

\section{Introduction}

Severe acute respiratory syndrome Coronavirus 2 (SARS-CoV-2, previously named 2019-nCoV) is a new coronavirus causing coronavirus disease 2019 (COVID-19) which first emerged in December 2019. ${ }^{1}$ As of 18 April, 2020, according to the World Health Organization (WHO), ${ }^{2} 2,160,207$ people all over the world have been infection-confirmed and 146,088 people have died. Human immunodeficiency virus (HIV) is another deadly virus and can cause acquired immunodeficiency syndrome (AIDS). According to the WHO, there are $\sim 37.9$ million people living with HIV. ${ }^{3}$ Rapid and early detection of these deadly viruses plays a critical role in facilitating early intervention and treatment, which, in turn, may reduce disease transmission risk.

Polymerase chain reaction (PCR) method is the most commonly used technology for pathogen nucleic acid detection and has been considered as a "gold standard" for infectious disease diagnostics due to its high sensitivity and specificity. 4, 5, 6 However, it typically relies on expensive equipment and well-trained personnel, all of which is not suitable for simple, rapid point of care (POC) diagnostic applications. In recent decades, several isothermal amplification methods, such as recombinase polymerase amplification (RPA) ${ }^{7}$ and loop- mediated isothermal amplification (LAMP) ${ }^{8}$, have been developed as attractive alternatives to conventional PCR method because of their simplicity, rapidity and low cost. However, there is still a challenge to apply them to develop accurate and reliable POC testing for clinical diagnostics due to undesired non-specific amplification signals (e.g., false-positive). ${ }^{9,} 10$

Recently, RNA-guided CRISPR/Cas nuclease-based nucleic acid detection has shown great promise for the development of next-generation point of care molecular diagnostics technology due to its high sensitivity, specificity and reliability. ${ }^{11,12}$ For example, several Cas nucleases, such as Cas12a, Cas12b 
and Cas13a, perform strong collateral cleavage activities in which the Cas nucleases activated by crRNA-

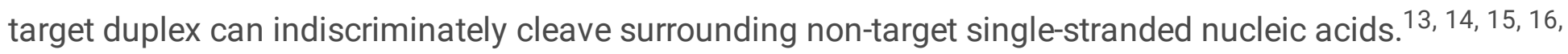
17 By combining with RPA pre- amplification, Cas13 and Cas12a nucleases have, respectively, been used to develop SHERLOCK (Specific High-sensitivity Enzymatic Reporter UnLOCKing) system ${ }^{18}$ and DETECTR (DNA Endonuclease-Targeted CRISPR Trans Reporter) system ${ }^{14}$ for highly sensitive and specific nucleic acid detection. Apart from the RPA pre-amplification method, some CRISPR-Cas-based nucleic acid detection utilized LAMP and PCR pre-amplification, such as CRISPR-Cas12b-assisted HOLMESv2 platform and SARS-CoV-2 DNA Endonuclease-Targeted CRISPR Trans Reporter (DETECTR). ${ }^{16}, 19$ However, these CRISPR- Cas-based nucleic acid detection methods typically require separate nucleic acid pre- amplification and multiple manual operations, which undoubtedly complicates the testing procedures and potentially increases the risk of carry-over contaminations due to amplification products transferring.

In this study, we report an All-m-One Dual CRISPR-Cas12a (termed "AIOD-CRISPR") assay for simple, rapid, ultrasensitive, specific, one-pot and visual detection of nucleic acids (DNA and RNA). Dual crRNAs are introduced to initiate highly sensitive dual CRISPR-based nucleic acid detection. In our AIOD-CRISPR assay, all components for nucleic acid amplification and CRISPR-based detection are thoroughly mixed in a single, one-pot reaction system and incubated at a single temperature (e.g., $37^{\circ} \mathrm{C}$ ), eliminating the need for separate pre- amplification and transfer of amplified product. As application examples, the AIODCRISPR assay was used to detect SARS-CoV $-2{ }^{20}$ and HIV-1 virus. ${ }^{21}$ Since the SARS-CoV-2 and HIV- 1 are retroviruses, we evaluated the performance of our AIOD-CRISPR assay by detecting both of their DNA and RNA. The AIOD-CRISPR method was clinically validated using COVID-19 patient samples and a lowcost hand warmer was directly used to as its incubator for instrument-free point of care diagnostics of COVID-19.

\section{Results}

AIOD-CRISPR assay system. As shown in Figure 1A, the AIOD-CRISPR assay system uses a pair of Cas12a-crRNA complexes generated by two individual crRNAs to bind two different sites which are close to the recognition sites of primers in the target sequence. The Cas12a- crRNA complexes are first prepared prior to being adding into the reaction solution containing RPA primers, SSDNA-FQ reporters, recombinase, single-stranded DNA binding protein (SSB), strand-displacement DNA polymerase, and target sequences. When incubating the AIOD- CRISPR reaction system in one pot at $\sim 37^{\circ} \mathrm{C}$, the RPA amplification is initiated and exposes the binding sites of the Cas12a-crRNA complexes due to the strand displacement. On one hand, when the Cas12a-crRNA complexes bind the target sites, the Cas12a endonuclease is activated and cleaves the SSDNA-FQ reporters, generating strong fluorescence signals. On the other hand, the amplified products generated during the RPA continuously trigger CRISPR-Cas12abased collateral cleavage activity. Previous studies ${ }^{14,17}$ have demonstrated that the collateral cleavage activity of the CRISPR-Cas12a system is independent of target strand cleavage. Therefore, target 
sequences for our AIOD-CRISPR assay are not limited by the Cas12a's protospacer adjacent motif (PAM) 22.

To systematically evaluate our AIOD-CRISPR assay system, we prepared and tested eight reaction systems (reactions \# 1-8) with various components (Figure 1B (i)).. The ssDNA-FQ reporter was a $5 \mathrm{nt}$ oligonucleotide (5'-TTATT-3') labelled by 5' 6-FAM (Fluorescein) fluorophore and $3^{\prime}$ lowa Black ${ }^{\circledR}$ FQ quencher. After incubation at $37^{\circ} \mathrm{C}$ for $40 \mathrm{~min}$, only reaction \# 5 containing target nucleic acid sequence, dual crRNAs, Cas12a, and RPA reaction mixture produced super-bright fluorescence signal (Figure 1B (i)), which could be directly visualized under a blue LED or UV light illuminator. Surprisingly, even under ambient light conditions without excitation, a color change from orange-yellow to green was directly observed in the reaction tube \# 5 by naked eyes. To further verify the specificity of the generated fluorescence signal, the assay products (self-probed fluorescence reporters) were subjected to denaturing polyacrylamide gel electrophoresis (PAGE). As shown in Figure 1B(ii), a strong band with shorter DNA size was observed only in the lane of reaction \# 5 , which resulted from the cleaved ssDNA-FQ reporters with strong fluorescence signal. In comparison, for other reaction systems, only weak bands with relatively longer DNA sizes were observed in their corresponding lanes, which may be attributed to fluorescence quench of the intact uncut ssDNA-FQ reporters. In addition, in real-time fluorescence curves, only reaction \# 5 showed a significantly increased fluorescence signal that saturated at $13 \mathrm{~min}$ (Figure 1B(iii)).. Thus, these results show that our AIOD-CRISPR assay provides a simple, rapid, one-pot approach for targetspecific nucleic acid detection.

Since a previous study reported that RPA amplification reaction is initiated after adding $\mathrm{MgOAc},{ }^{23}$ we are interested in knowing if nucleic acid amplification is efficiently initiated at room temperature during sample preparation in our AIOD-CRISPR assay system. We prepared two AIOD-CRISPR solutions (one positive and one negative) and allowed them to remain at room temperature for $10 \mathrm{~min}$. As shown in Figure S1, no significant fluorescence change between positive and negative samples was observed in the AIOD-CRISPR systems at room temperature. In comparison, there was an obvious fluorescence increase after just one-minute incubation at $37^{\circ} \mathrm{C}$ (Figure S1).. Eventually, the fluorescence signal was saturated after 15 -min incubation at $37^{\circ} \mathrm{C}$. Therefore, our AIOD-CRISPR assay system is mainly triggered after reaction temperature is elevated to $\sim 37^{\circ} \mathrm{C}$.

Optimization of AIOD-CRISPR assay. Collateral cleavage of the SSDNA-FQ reporters by the Cas12a nuclease is triggered by the binding of crRNA to target sites. ${ }^{13,14}$ Here, we hypothesize that more binding opportunities can increase the collateral cleavage activity and eventually improve the detection sensitivity. To test our hypothesis, we designed a pair of crRNAs to respectively recognize two different target sites in our AIOD-CRISPR assay. A pUCIDT-AMP plasmid containing 300 bp HIV-1 p24 gene cDNA (p24 plasmid) was used as the target and three different design strategies for primers and crRNAs were investigated (Figure 2A).. As shown in Figure 2B, the AIOD-CRISPR with dual crRNAs (crRNA1+crRNA2) showed slightly higher fluorescence signals compared to that with single crRNA2, but much better than that with single crRNA1. In addition, doubling the amount of either crRNA1 or crRNA2 did not benefit the detection efficiency. Furthermore, we evaluated and compared the detection sensitivity of the AIOD- 
CRISPR system with dual crRNAs and single crRNA. As shown in Figure $2 C$ and $D$, the AIOD-CRISPR with dual crRNAs was able to consistently detect as low as 1.2 copies of the p24 plasmid templates with improved fluorescence, while the AIOD- CRISPR assay with single crRNA2 did not. Thus, by introducing dual crRNAs into the AIOD- CRISPR assay, it does not only increase fluorescence signals, but also improve the detection sensitivity.

We further optimized ssDNA-FQ reporters in our AIOD-CRISPR assay because the reporter concentration plays a crucial role in fluorescence readout. As shown in Figure $S 2 A$, the higher the concentration of the ssDNA-FQ reporters, the stronger the fluorescence signal and the shorter the threshold time. As to threshold time and visual detection, the minimal concentration for saturated values was $4 \mu \mathrm{M}$ (Figure $S 2 B-S 2 D)$.. Collateral cleavage efficiency of the activated Cas12a nuclease represents an ability to cut SsDNA-FQ reporters around it. ${ }^{13,14}$ Thus, increasing the ssDNA-FQ reporter concentration can improve the fluorescence signals. In addition, we also investigated the effect of the primer concentration on the AIODCRISPR assay. As shown in Figure S3, the optimal concentration of the primers was $0.32 \mu \mathrm{M}$. Together, introducing dual crRNAs with an increased ssDNA-FQ reporter concentration enables highly efficient AIOD-CRISPR assay.

HIV- 1 detection by AIOD-CRISPR assay. To investigate the sensitivity of the AIOD-CRISPR assay for HIV1 DNA detection, we first applied the optimized AIOD-CRISPR assay to detect various copies of HIV-1 p24 plasmid templates (from $1.2 \times 10^{0}$ to $1.2 \times 10^{5}$ copies). As shown in Figure $3 A$, the AIOD-CRISPR could consistently detect as low as 1.2 copies HIV-1 p24 plasmid DNA in both real-time and endpoint visual detection, which was further verified by the denaturing PAGE. Although incubated for $40 \mathrm{~min}$, the AIODCRISPR assay could detect and identify 1.2 copies of HIV-1 DNA in just 1-min incubation based on the endpoint fluorescence intensity (Figure S4),, which shows that our AIOD-CRISPR assay provides a superfast (few minutes) and ultrasensitive (several copies) detection of nucleic acids.

Next, we applied the AIOD-CRISPR assay to detect HIV-1 RNA sequence by adding Avian Myeloblastosis Virus (AMV) Reverse Transcriptase, namely reverse transcription AIOD- CRISPR (RT-AIOD-CRISPR). The HIV RNA target is a 1057-nt fragment of gag (p24 included) gene prepared using T7 promotor-tagged RTPCR and T7 RNA polymerase-based transcription (Figure S5A).. The detection region of the AIOD-CRISPR assay was further verified by Sanger sequencing (Figure S5B).. To achieve highly sensitive HIV-1 RNA detection, we optimized the AMV concentration. As shown in Figure S5C, the RT-AIOD-CRISPR performed the highest efficiency with $0.32 \mathrm{U} / \mu \mathrm{L}$ AMV when incubated at $37^{\circ} \mathrm{C}$. Furthermore, we investigated the detection sensitivity of our RT-AIOD-CRISPR assay using $1.1 \times 10^{0}, 1.1 \times 10^{1}, 1.1 \times 10^{3}$, and $1.1 \times 10^{5}$ copies of HIV-1 gag RNA templates. Figure $3 B$ showed that the RT-AIOD-CRISPR assay was able to consistently detect 11 copies of HIV-1 RNA targets in both real-time and endpoint fluorescence visual detections. However, visual detection without excitation light can only identify $1.1 \times 10^{5}$ copies of HIV-1 RNA targets. Additionally, the RT- AIOD-CRISPR's sensitivity was further confirmed by denaturing PAGE analysis (Figure $3 B)$.. 
In addition to detecting artificial HIV-1 gag RNA, we also evaluated the RT-AIOD-CRISPR's performance using HIV-1 RNA extracted from human plasma samples. As shown in Figure 3C, real-time RT-AIODCRISPR assay was able to detect 500 copies of HIV viral RNA within less than 20 min. However, visual RTAIOD-CRISPR detection took relatively long incubation time (up to $90 \mathrm{~min}$ ) to achieve the similar sensitivity (Figure S6A).. The reduced sensitivity of the RT-AIOD-CRISPR for extracted HIV RNA detection may be attributed to either potential inhibitors in the extracts or RNA degradation during extraction. Despite this, the real-time AIOD-CRISPR assay showed a comparable sensitivity compared to real-time RTPCR assay (Figure S6B)..

SARS-CoV-2 detection by AIOD-CRISPR assay. As shown in Figure 4A, a pUCIDT-AMP plasmid containing $316 \mathrm{nt}$ SARS-CoV-2 N gene cDNA (N plasmid) was first prepared as the target to develop our AIODCRISPR assay. Figure $4 B$ shows that our AIOD-CRISPR assay could detect 1.3 copies of SARS-CoV-2 N plasmids in both real-time and visual detections, offering a rapid and nearly single-molecule level sensitive detection. To evaluate the detection specificity, we tested our AIOD-CRISPR assay using commercially available control plasmids containing the complete $\mathrm{N}$ gene from SARS-CoV-2 (SARS-CoV2 _PC, Catalogue \# 10006625, IDT), SARS (SARS-CoV_PC, Catalogue \# 10006624, IDT), and Middle East respiratory syndrome (MERS) (MERS-CoV (Middle East respiratory syndrome coronavirus)_PC, Catalogue \# 10006623, IDT), as well as the Hs_RPP30 control (Hs_RPP30_PC, Catalogue \# 10006626, IDT) with a portion of human RPP30 gene. Figure $4 C$ shows that only the reaction with SARS-CoV-2_PC had the positive signal in both real- time and visual detections, demonstrating that our developed AIOD-CRISPR assay possesses high specificity without cross reactions for non-SARS-CoV-2 targets.

Next, we used T7 promotor-tagged PCR and T7 RNA polymerase to prepare SARS-CoV-2 N gene RNA sequences to develop the RT-AIOD-CRISPR assay (Figure S7A).. The detection region of the RT-AIODCRISPR was verified by Sanger sequencing (Figure 7B).. As shown in Figure 4D, the RT-AIOD-CRISPR assay could consistently detect down to 4.6 copies of SARS-CoV-2 N RNA targets in both real-time and visual detections. In addition, Figure $4 E$ shows that the endpoint fluorescence intensity after 1 min RTAIOD-CRISPR reaction was able to identify 4.6 copies of SARS-CoV-2 N RNA. Therefore, our AIODCRISPR assay provides an ultrarapid, highly sensitive and specific method for SARS-CoV-2 detection.

To further demonstrate its point of care diagnostic application, we used a low-cost hand warmer $(\sim \$ 0.3$ per bag) as the incubator of our AIOD-CRISPR assay and detect COVID-19 patient samples. As shown in Figure $6 A$, the AIOD-CRISPR assay tubes were directly placed on an air-activated hand warmer without need for any electric incubator. The endpoint fluorescence result can be observed by the naked eye under LED light. Figure $6 B$ shows that two SARS-CoV-2-positive samples incubated in the hand warmer bag were visually detected and identified within as short as $20 \mathrm{~min}$. The longer the incubation time, the stronger the fluoscence signal of the positive samples. Additionally, a similar result was achieved through analyzing the green value of the fluorescence images using the ImageJ software (Figure 6C).. Therefore, our AIOD-CRISPR method provides a simple, rapid and visual approach for SARS- CoV-2 detection and has the potential to develop an instrument-free point of care diagnostics of the COVID-19. 


\section{Discussion}

The emergence of the new coronavirus SARS-CoV-2, and its rapid spread through many countries, has been labeled as a global health emergency by the WHO. ${ }^{24} \mathrm{HIV}$ is another deadly retrovirus that has caused deaths of 32 million people since the beginning of the epidemic. ${ }^{3}$ Early diagnosis of these severe infections is crucial to prevent the rapid spread of these deadly viruses globally. Nucleic acid amplification testing (e.g., PCR/RT-PCR) represents the most sensitive and specific method for the early detection of the pathogens, ${ }^{25}, 26$ but current PCR technology is not suitable for rapid point of care diagnostic application due to the need for specialized laboratory equipment and trained technicians. The limitations of current detection technology represent serious barriers for the real-time monitoring and detection of these highly contagious pathogens to prevent them spreading from person-to- person. Thus, there is an urgent need for a simple, easy-to-use, and inexpensive diagnostic approach.

In this study, we described a simple, rapid, ultrasensitive and highly specific AIOD-CRISPR assay for the detection of the SARS-CoV- 2 and HIV- 1 virus. This AIOD-CRISPR assay method is, to the best of our knowledge, the first system that allows all components to be incubated in one pot for CRISPR-based nucleic acid detection, enabling simple, all-in-one molecular diagnostics without need for separate and complex manual operations. The AIOD- CRISPR assay takes advantage of dual CRISPR-Cas12a-based detection strategy to improve the detection sensitivity and fluorescence signals. Importantly, the detection results of the AIOD-CRISPR assay can be directly visualized by the naked eye, significantly simplifying the detection process and eliminating the need for separate lateral flow-based detection ${ }^{19}$.

Compared to previously reported CRISPR-based nucleic acid detection methods, 14, 16, 18, 19, 27, 28, 29 our versatile and robust AIOD-CRISPR assay has some distinctive advantages and provides a true single reaction system. In our AIOD-CRISPR assay, the components for both isothermal amplification and CRISPR-based detection are prepared in one-pot, completely circumventing the separate pre-amplification of target nucleic acids, ${ }^{14}$ or physical separation of Cas enzyme. ${ }^{29}$ The AIOD-CRISPR assay enables superfast (few minutes), ultrasensitive (few copies), and highly specific nucleic acid detection. With our AIODCRISPR assay, we were able to detect as low as 1.3 copies of DNA targets and 4.6 copies of RNA targets in SARS-CoV-2 detection. Although we incubated our assay system at $37^{\circ} \mathrm{C}$ for $40 \mathrm{~min}$ in our experiment, we demonstrated that the AIOD-CRISPR assay could detect and identify 1.2 copies of HIV-1 DNA and 4.6 copies of SARS-CoV-2 N RNA after just 1-minute incubation (Figure $S 4 A$ and $4 E$ ).. We attribute the superfast testing speed and ultra-high detection sensitivity of our AIOD-CRISPR assay to: i) the introduction of unique dual CRISPR-Cas12a detection methodology, ii) the increased concentration of SsDNA-FQ reporters, and iii) the combination of RPA amplification and CRISPR-Cas12a-based detection. Also, the AIOD-CRISPR assay showed a high specificity in the SARS-CoV-2 detection without any crossinteraction (false positives) with other sequences (e.g., SARS-CoV, MERS-CoV) (Figure 4C),, which may be due to the nature of the CRIPSR-Cas12a's single-base specificity ${ }^{30,31 .}$

Although in this manuscript, we demonstrated only qualitative detection of nucleic acids, we anticipate that our method is able to achieve semi-quantitative detection by reducing its incubation time and 
quantifying endpoint fluorescence intensities. Indeed, as shown in Figure S4A, we demonstrated the feasibility of the semi-quantitative detection of the HIV DNA by incubating the AIOD-CRISPR reaction system for 1 minute. In addition, by adding AMV reverse transcriptase, the AIOD-CRISPR assay can be easily developed as one-step RT- AIOD-CRISPR assay to detect RNA targets such as HIV- 1 and SARSCoV-2 RNAs, which facilitates the CRISPR-Cas12a-based RNA detection without need for the separate preparation of cDNA. To evaluate the validity and clinical applications of our AIOD-CRISPR assay, we adapted it to detect HIV-1 virus in human plasma samples and SARS-CoV-2 virus in nasal swab samples, achieving consistent detection results with that of RT-PCR method. Most importantly, we successfully demonstrated an instrument-free AIOD-CRISPR assay for SARS-CoV-2 detection in clinical samples by using a simple hand warmer.

Further improvement and development are to integrate our AIOD-CRISPR assay into a disposable microfluidics chip platform, 32, 33,34,35 enabling fully-integrated, "sample to result", multiplexed detection. On one hand, all reagents of the AIOD-CRISPR assay can be lyophilized and pre-stored in a disposable microfluidic chamber, ${ }^{36}$ which eliminates need for cold chains and enables rapid detection outside of a laboratory setting. On the other hand, multiplexing detection can be developed by combining multiplexed microfluidics technology. ${ }^{37}, 38,39$ Symptom of COVID-19 is non-specific and similar to other respiratory illnesses. ${ }^{40}$ Therefore, to enable effective disease treatment and management, it is critical to simultaneously detect and differentiate SARS-CoV-2 and other viral infections (e.g., SARS- CoV, MERSCoV) by microfluidic-based multiplexed detection with single sample.

Since our AIOD-CRISPR assay generates strong fluorescence signals at the endpoint, it is possible to record, analyze and report the detection results by taking advantage of ubiquitous smartphone technology. $41,42,43$ The smartphone can be programmed to take fluorescence photos, convert the images into fluorescence intensity, analyze the data, and report the qualitative/semi-quantitative test results. Further, the test results can be wirelessly transmitted to a website or remote server ${ }^{42}$ and made available together with GPS coordinates to the patient's doctor and public health officials. This is critical to allow simple, rapid, smart, connected disease diagnostics and tracking.

In summary, the AIOD-CRISPR assay has been demonstrated to be a rapid, all-in-one, isothermal approach for nucleic acid (DNA and RNA) detection with nearly single-molecule level sensitive and single-base specificity. In turn, such simple and robust method has great potential in the future development of a next-generation point of care molecular diagnostics technology for the rapid detection of infectious diseases (e.g., COVID-19) at home or in small clinics.

\section{Methods}

Materials. Oligonucleotides (primers), ssDNA-FQ reporters, pUCIDT (Amp) plasmids with customized gene sequences, and the control plasmids containing the complete $\mathrm{N}$ gene from SARS-CoV-2, SARS, and MERS, as well as the Hs_RPP30 control were synthesized by or purchased from Integrated DNA Technologies (Coralville, IA). AcroMetrix ${ }^{\text {TM }}$ HIV-1 plasma control samples, dNTP Set (100 mM), Gel 
Loading Buffer II (Denaturing PAGE), PureLink ${ }^{T M}$ Quick Gel Extraction Kit and TURBO DNA-free ${ }^{\text {TM }}$ Kit were purchased from Thermo Fisher Scientific (Waltham, MA). EvaGreen ${ }^{\circledR}$ dye (20x) was purchased from Biotium (Fremont, CA). TEMED, $\left(\mathrm{NH}_{4}\right)_{2} \mathrm{~S}_{2} \mathrm{O}_{8}, 30 \%$ acrylamide/bis-acrylamide solution, 10× TBE Buffer, and SsoAdvanced ${ }^{\text {TM }}$ Universal SYBR ${ }^{\circledR}$ Green PCR Supermix were purchased from Bio-Rad Laboratories (Hercules, CA). EnGen ${ }^{\circledR}$ Lba Cas12a (Cpf1) $(100 \mu \mathrm{M})$, deoxynucleotide (dNTP) mix (10 mM of each), and Avian Myeloblastosis Virus (AMV) Reverse Transcriptase (10,000 units/mL) were purchased from New England BioLabs (Ipswich, MA). QIAamp ${ }^{\circledR}$ Viral RNA Mini Kit, RNeasy ${ }^{\circledR}$ MinElute ${ }^{\text {TM }}$ Cleanup Kit, and QIAGEN $^{\circledR}$ OneStep RT-PCR Kit were purchased from QIAGEN (Frederick, MD). RiboMAX ${ }^{\mathrm{TM}}$ Large Scale RNA Production Systems-T7 was purchased from Sigma-Aldrich (St. Louis, MO). TwistAmp ${ }^{\circledR}$ Liquid Basic Kit was purchased from TwistDx ${ }^{T M}$ Limited (Maidenhead, UK). The LED blue light illuminator (Maestrogen UltraSlim) was purchased from Fisher Scientific (Pittsburgh, PA). Eight SARS-CoV-2 clinical samples were collected through swab sampling and their viral RNAs were extracted by utilizing $140 \mu \mathrm{L}$ of each sample and eluting with $140 \mu \mathrm{L}$ of buffer of the QIAamp DSP Viral RNA Mini Kit (QIAGEN N. V., Venlo, The Netherlands). These eight samples were screened for SARS- COV-2 by CDC-approved RT-PCR assays (Thermo Fisher Scientific Inc., Waltham, MA) prior to our AIOD-CRISPR assays.

AIOD-CRISPR assays. The AIOD-CRISPR system was prepared separately as Component A, B and C. Component A contained 1× Reaction Buffer, 1× Basic E-mix, $14 \mathrm{mM} \mathrm{MgOAc}, 320 \mathrm{nM}$ each of primers, and $1.2 \mathrm{mM}$ dNTPs. Component B consisted of $4 \mu \mathrm{M}$ of ssDNA-FQ reporters and $1 \times$ Core Reaction Buffer. Component $\mathrm{C}$ was the Cas12a-crRNA mix with $0.64 \mu \mathrm{M}$ each of crRNAs and $1.28 \mu \mathrm{M} \mathrm{EnGen}{ }^{\circledR} \mathrm{Lba}$ Cas12a. The concentration in each component was calculated based on the finally assembled $25-\mu \mathrm{L}$ AIOD-CRISPR system. In a typical AIOD-CRISPR assay, $1 \mu \mathrm{L}$ of the target solution was mixed with $20 \mu \mathrm{L}$ of Component $\mathrm{A}$ and

$2.5 \mu \mathrm{L}$ of Component B. This assembled mixture was then mixed with $1.5 \mu \mathrm{L}$ of Component $\mathrm{C}$ to form final $25 \mu \mathrm{L}$ of AIOD-CRISPR system. For RT-AIOD-CRISPR assays, most components were the same as those in the AIOD-CRISPR system above, except supplementing $0.32 \mathrm{U} / \mu \mathrm{L}$ AMV Reverse Transcriptase in Component A. Real-time fluorescence detection was carried out in the Bio-Rad CFX96 Touch $^{\text {TM }}$ Real-Time PCR Detection System. Visual detection was accomplished through imaging the tubes in the LED blue

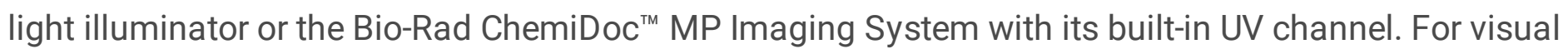
detection based on the reaction solution's color change, $8 \mu \mathrm{M}$ of ssDNA-FQ reporters should be used. All the reactions were incubated at $37^{\circ} \mathrm{C}$ for $40 \mathrm{~min}$ or the denoted time in figures. The endpoint fluorescence was the raw fluorescence determined by the Real-Time PCR Detection System. The unpaired t-test was applied for the statistical analysis in GraphPad Software Prism 8. A saturated fluorescence intensity was the maximum intensity which the Real-Time PCR Detection System could determine. After reaction, the AIOD-CRISPR solution was mixed with isometric Gel Loading Buffer II prior to 15\% denaturing PAGE with $8 \mathrm{M}$ urea and gel imaging in the Imaging System.

In vitro RNA preparation using T7 RNA polymerase. For HIV-1 gag RNA, OneStep RT- PCR composed of 1× QIAGEN OneStep RT-PCR Buffer, $0.4 \mathrm{mM}$ of each dNTP, $0.6 \mu \mathrm{M}$ of each primer, $2.0 \mu \mathrm{L}$ of QIAGEN 
OneStep RT-PCR Enzyme Mix, and $1.0 \mu \mathrm{L}$ of the viral RNA extracted from HIV-1 plasma control was conducted to amplify the $1057 \mathrm{nt}$ gag sequence. The thermal cycling protocol included $30 \mathrm{~min}$ at $50^{\circ} \mathrm{C}$ for reverse transcription, $15 \mathrm{~min}$ at $95^{\circ} \mathrm{C}$ for initial PCR activation, 35 cycles of the 3 -step cycling $(1 \mathrm{~min}$ at $94^{\circ} \mathrm{C}$ for denaturation, $1 \mathrm{~min}$ at $55^{\circ} \mathrm{C}$ for annealing, and $1 \mathrm{~min}$ at $72^{\circ} \mathrm{C}$ for extension), and $10 \mathrm{~min}$ at $50^{\circ} \mathrm{C}$ for final extension. For SARS-CoV-2 N RNA, the PCR system contained 1× SsoAdvanced ${ }^{\mathrm{TM}}$ Universal SYBR ${ }^{\circledR}$ Green PCR Supermix, $0.4 \mu \mathrm{M}$ of each primer, and $1.0 \mu \mathrm{L}$ of $1.2 \times 10^{5}$ copies/ $\mu \mathrm{L}$ HIV -1 p24 plasmid solution was used to amplify the $316 \mathrm{nt} \mathrm{N}$ sequence. The thermal cycling was $2.5 \mathrm{~min}$ at $98^{\circ} \mathrm{C}$ for initial denaturation, 35 cycles of $15 \mathrm{~s}$ at $95^{\circ} \mathrm{C}$ for denaturation and $30 \mathrm{~s}$ at $60^{\circ} \mathrm{C}$ for annealing and extension. The products of PCR/RT-PCR were all confirmed by agarose gel electrophoresis and Sanger sequencing. Afterwards, the products with the accurate sizes were extracted and purified using the Gel Extraction Kit. In vitro transcription was achieved through incubating the reaction system containing $8 \mu \mathrm{L}$ of $5 \times \mathrm{T} 7$ Transcription Buffer, $3 \mu \mathrm{L}$ each of 100 mM rNTPs, $4 \mu \mathrm{L}$ of the Enzyme Mix with T7 RNA polymerase, 16 $\mu \mathrm{L}$ of the gel- extracted PCR/RT-PCR products at $37^{\circ} \mathrm{C}$ for $4 \mathrm{~h}$. Then, the transcription products were treated by DNase (from the TURBO DNA-free TM Kit) to degrading the DNA and the RNA was extracted and purified using the RNeasy@ MinElute ${ }^{\mathrm{TM}}$ Cleanup Kit. The purity and concentration of the collected RNA were determined using NanoDrop ${ }^{\text {TM }}$ One/One ${ }^{\mathrm{C}}$ Microvolume UV-Vis Spectrophotometry (Thermo Fisher Scientific).

OneStep RT-PCR assay. QIAGEN ${ }^{\circledR}$ OneStep RT-PCR Kit was used for the RT-PCR assay. The primers (FP: 5'ATTATCAGAAGGAGCCACC-3'; RP: 5'-CATCCTATTTGTTCCTGAAGG-3') for HIV-1 RNA detection were obtained from the reported literature. ${ }^{44}$ According to the instruction manual, the OneStep RT-PCR assay $(50 \mu \mathrm{L})$ contained 1× QIAGEN OneStep RT- PCR Buffer, $400 \mu \mathrm{M}$ of each dNTP, $600 \mathrm{nM}$ each of primers (FP and RP), 2.0 $\mu \mathrm{L}$ of QIAGEN OneStep RT-PCR Enzyme Mix, 0.8× EvaGreen ${ }^{\circledR}$ dye, and $5.0 \mu \mathrm{L}$ of the RNA template solution. The thermal cycling protocol included $30 \mathrm{~min}$ at $50^{\circ} \mathrm{C}$ for reverse transcription, $15 \mathrm{~min}$ at $95^{\circ} \mathrm{C}$ for initial PCR activation step, 35 cycles of the 3 -step cycling (30 s at $94^{\circ} \mathrm{C}$ for denaturation, $30 \mathrm{~s}$ at $55^{\circ} \mathrm{C}$ for annealing, and $1 \mathrm{~min}$ at $72^{\circ} \mathrm{C}$ for extension), and $10 \mathrm{~min}$ at $72^{\circ} \mathrm{C}$ for final extension, followed by the melt-curve analysis (from $65^{\circ} \mathrm{C}$ to $95^{\circ} \mathrm{C}$ with $0.5^{\circ} \mathrm{C}$ increment). Real-time OneStep RT-PCR assay was conducted in the CFX96 Touch $^{\text {TM }}$ Real-Time PCR Detection System and the plate read was set at the annealing in the 3-step cycling.

\section{Declarations}

\section{Acknowledgements}

The work was supported, in part, by R01EB023607, R01CA214072, and R21TW010625.

\section{Competing Interests}

The authors declare there are no competing interests.

\section{References}


1. Zhu N, et al. A novel coronavirus from patients with pneumonia in China, 2019. New England Journal of Medicine, (2020).

2. Coronavirus disease 2019 (COVID-19) Situation Report-89. https://www.who.int/docs/defaultsource/coronaviruse/situation-reports/20200418-sitrep-89-covid-19.pdf. (2020).

3. HIV/AIDS data and statistics. https://www.who.int/hiv/data/en/. (2019).

4. Radmard S, et al. Clinical utilization of the FilmArray meningitis/encephalitis (ME) multiplex polymerase chain reaction (PCR) assay. Frontiers in neurology 10, 281 (2019).

5. Cao L, et al. Advances in digital polymerase chain reaction (dPCR) and its emerging biomedical applications. Biosens Bioelectron 90, 459-474 (2017).

6. Wang AM, Doyle MV, Mark DF. Quantitation of mRNA by the polymerase chain reaction. Proceedings of the National Academy of Sciences 86, 9717-9721 (1989).

7. Piepenburg 0 , Williams $\mathrm{CH}$, Stemple DL, Armes NA. DNA detection using recombination proteins. PLoS biology 4, (2006).

8. Notomi T, et al. Loop-mediated isothermal amplification of DNA. Nucleic Acids Res $\mathbf{2 8}$, e63-e63 (2000).

9. Tian B, Minero GAS, Fock J, Dufva M, Hansen CRISPR-Cas12a based internal negative control for nonspecific products of exponential rolling circle amplification. Nucleic Acids Res 48, e30-e30 (2020).

10. Rolando JC, Jue E, Barlow JT, Ismagilov RF. Real-time kinetics and high-resolution melt curves in single-molecule digital LAMP to differentiate and study specific and non-specific amplification. Nucleic Acids Res, (2020).

11. Li Y, Li S, Wang J, Liu G. CRISPR/Cas systems towards next-generation Trends Biotechno/37, 730743 (2019).

12. Chertow DS. Next-generation diagnostics with CRISPR. Science 360, 381-382 (2018).

13. Li S-Y, Cheng Q-X, Liu J-K, Nie X-Q, Zhao G-P, Wang J. CRISPR-Cas12a has both cis- and transcleavage activities on single-stranded DNA. Cell research 28, 491-493 (2018).

14. Chen JS, et al. CRISPR-Cas12a target binding unleashes indiscriminate single-stranded DNase activity. Science $\mathbf{3 6 0}$, 436-439 (2018).

15. Abudayyeh 00 , et al. $\mathrm{C} 2 \mathrm{c} 2$ is a single-component programmable RNA-guided RNA-targeting CRISPR effector. Science 353, aaf5573 (2016).

16. Li L, et al. HOLMESv2: a CRISPR-Cas12b-assisted platform for nucleic acid detection and DNA methylation quantitation. ACS synthetic biology 8, 2228-2237 (2019).

17. Jeon Y, et al. Direct observation of DNA target searching and cleavage by CRISPR- Cas12a. Nature communications 9, 1-11 (2018).

18. Gootenberg JS, et al. Nucleic acid detection with CRISPR-Cas13a/C2c2. Science 356, 438-442 (2017).

19. Broughton JP, et al. CRISPR-Cas12-based detection of SARS-CoV-2. Nat Biotechnol, 1-5 (2020). 
20. Gorbalenya $A E$, et al. The species Severe acute respiratory syndrome-related coronavirus: classifying 2019-nCoV and naming it SARS-CoV-2. Nature Microbiology, (2020).

21. Rhee S-Y, et al. Trends in the molecular epidemiology and genetic mechanisms of transmitted human immunodeficiency virus type 1 drug resistance in a large US clinic population. Clinical Infectious Diseases 68, 213-221 (2019).

22. Yang $\mathrm{H}$, Gao P, Rajashankar KR, Patel DJ. PAM-dependent target DNA recognition and cleavage by C2c1 CRISPR-Cas endonuclease. Cell 167, 1814-1828. e1812 (2016).

23. Yeh E-C, Fu C-C, Hu L, Thakur R, Feng J, Lee LP. Self-powered integrated microfluidic point-of-care low-cost enabling (SIMPLE) chip. Science advances 3, e1501645 (2017).

24. Updated WHO recommendations for international traffic in relation to COVID-19 outbreak. https://www.who.int/news-room/articles-detail/updated-who-recommendations-for-internationaltraffic-in-relation-to-covid-19-outbreak. (2020).

25. Kralik P, Ricchi M. A basic guide to real time PCR in microbial diagnostics: definitions, parameters, and everything. Frontiers in microbiology 8, 108 (2017).

26. Espy $M$, et al. Real-time PCR in clinical microbiology: applications for routine laboratory testing. Clinical microbiology reviews 19, 165-256 (2006).

27. Cong L, et al. Multiplex genome engineering using CRISPR/Cas systems. Science $\mathbf{3 3 9}$, 819-823 (2013).

28. Zhou W, Hu L, Ying L, Zhao Z, Chu PK, Yu X-F. A CRISPR-Cas9-triggered strand displacement amplification method for ultrasensitive DNA detection. Nature communications 9, 1-11 (2018).

29. Wang B, et al. Cas12aVDet: a CRISPR/Cas12a-based platform for rapid and visual nucleic acid detection. Anal Chem 91, 12156-12161 (2019).

30. Strohkendl I, Saifuddin FA, Rybarski JR, Finkelstein IJ, Russell R. Kinetic basis for DNA target specificity of CRISPR-Cas12a. Molecular cell 71, 816-824. e813 (2018).

31. Kim H, et al. Enhancement of Target Specificity of CRISPR-Cas12a by Using a Chimeric DNA-RNA Guide. bioRxiv, (2020).

32. Song J, Mauk MG, Hackett BA, Cherry S, Bau HH, Liu C. Instrument-free point-of-care molecular detection of Zika virus. Anal Chem 88, 7289-7294 (2016).

33. Chen $D$, et al. An integrated, self-contained microfluidic cassette for isolation, amplification, and detection of nucleic acids. Biomed Microdevices 12, 705-719 (2010).

34. Park S, Zhang Y, Lin S, Wang T-H, Yang S. Advances in microfluidic PCR for point-of- care infectious disease diagnostics. Biotechnol Adv 29, 830-839 (2011).

35. Ferguson BS, et al. Genetic analysis of $\mathrm{H} 1 \mathrm{~N} 1$ influenza virus from throat swab samples in a microfluidic system for point-of-care diagnostics. J Am Chem Soc 133, 9129-9135 (2011).

36. Song J, Liu C, Mauk MG, Peng J, Schoenfeld T, Bau HH. A multifunctional reactor with dry-stored reagents for enzymatic amplification of nucleic acids. Anal Chem 90, 1209- 1216 (2018). 
37. Fang $X$, Chen $\mathrm{H}, \mathrm{Yu}$ S, Jiang $X$, Kong J. Predicting viruses accurately by a multiplex microfluidic loopmediated isothermal amplification chip. Anal Chem 83, 690-695 (2011).

38. Dou M, Sanjay ST, Dominguez DC, Liu P, Xu F, Li X. Multiplexed instrument-free meningitis diagnosis on a polymer/paper hybrid microfluidic biochip. Biosens Bioelectron 87, 865-873 (2017).

39. Song J, et al. Two-stage isothermal enzymatic amplification for concurrent multiplex molecular detection. Clin Chem 63, 714-722 (2017).

40. Wu D, Wu T, Liu Q, Yang The SARS-CoV-2 outbreak: what we know. International Journal of Infectious Diseases, (2020).

41. Chen W, et al. Mobile platform for multiplexed detection and differentiation of disease- specific nucleic acid sequences, using microfluidic loop-mediated isothermal amplification and smartphone detection. Anal Chem 89, 11219-11226 (2017).

42. Song J, et al. Smartphone-based mobile detection platform for molecular diagnostics and spatiotemporal disease mapping. Anal Chem 90, 4823-4831 (2018).

43. Yin $\mathrm{K}$, et al. Synergistically enhanced colorimetric molecular detection using smart cup: a case for instrument-free HPV-associated cancer screening. Theranostics 9, 2637 (2019).

44. Curtis KA, Rudolph DL, Owen SM. Rapid detection of HIV-1 by reverse-transcription, loop-mediated isothermal amplification (RT-LAMP). Journal of virological methods 151, 264-270 (2008).

\section{Figures}



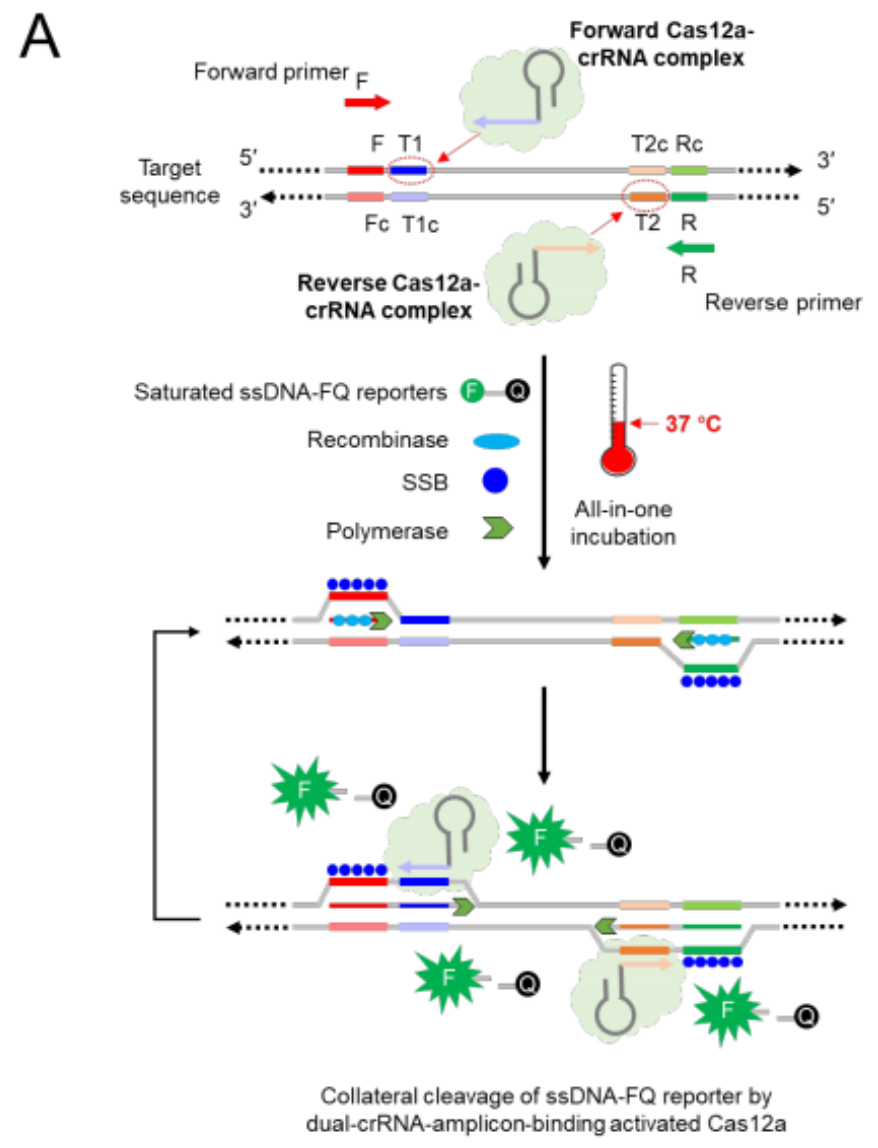

B

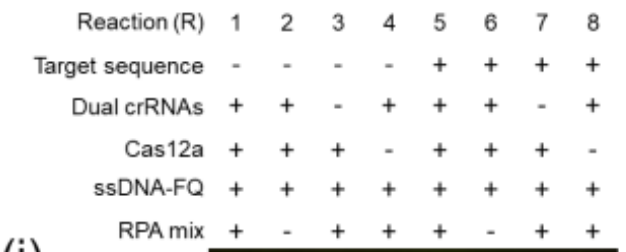

(i)

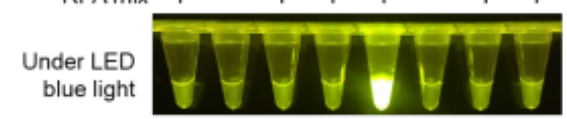

Under UV light

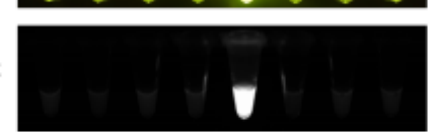

(ii)

No excitation

light
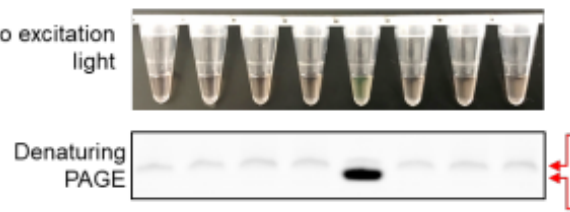

Uncut ssDNAFQ reporter

(iii)

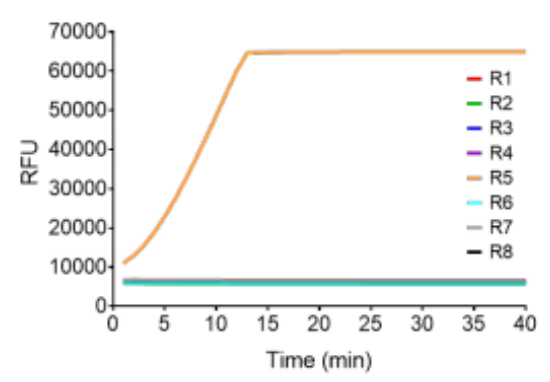

Cleaved ssDNAFQ reporter

\section{Figure 1}

Design and working principle of the AIOD-CRISPR assay. (A) Schematic of the AIOD-CRISPR assay system. SSB, single-stranded DNA binding protein. (B) Development and evaluation of the AIOD-CRISPR assay system. The ssDNA-FQ reporter was labelled by 5' 6-FAM (Fluorescein) fluorophore and 3' lowa Black $\AA F Q$ quencher. Recombinase polymerase amplification (RPA) mix from TwistAmp $\AA$ Liquid Basic kit was composed of 1× Reaction Buffer, 1× Basic E-mix, 1× Core Reaction Buffer, 14 mM MgOAc, $320 \mathrm{nM}$ each of primers and $1.2 \mathrm{mM}$ dNTPs. Dual crRNAs contained $0.64 \mu \mathrm{M}$ each of crRNAs. HIV-1 p24 plasmid (1.2× 105 copies), $8 \mu \mathrm{M}$ of ssDNA-FQ reporters, and $1.28 \mu \mathrm{M}$ EnGen ${ }^{\circledR}$ Lba Cas12a (Cpf1) were used. (i) Eight reaction systems with various components and their endpoint images after 40-min incubation. (ii) Denaturing PAGE analysis of the AIOD-CRISPR products. (iii) Real-time fluorescence detection of the AIOD-CRISPR assay for eight reaction systems. 


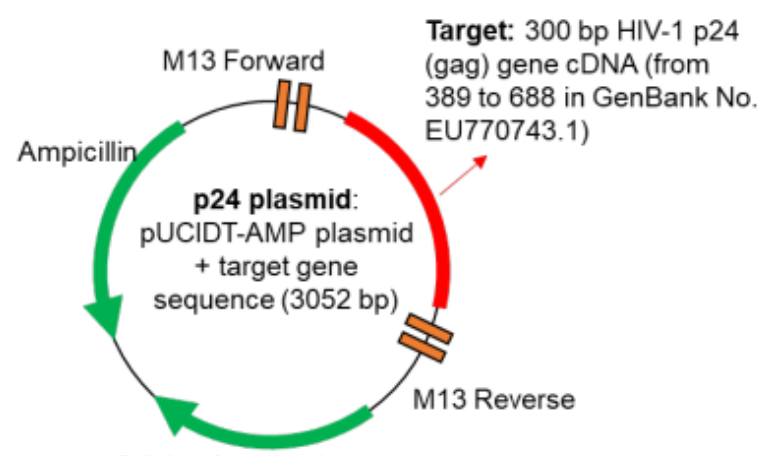

Origin of replication

B

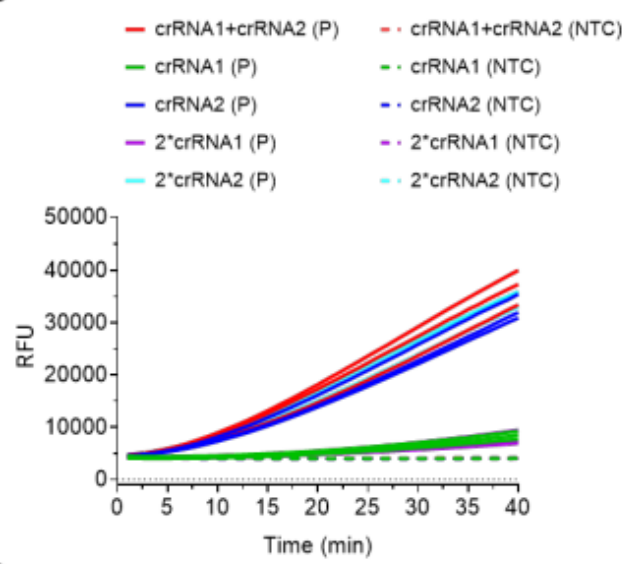

D

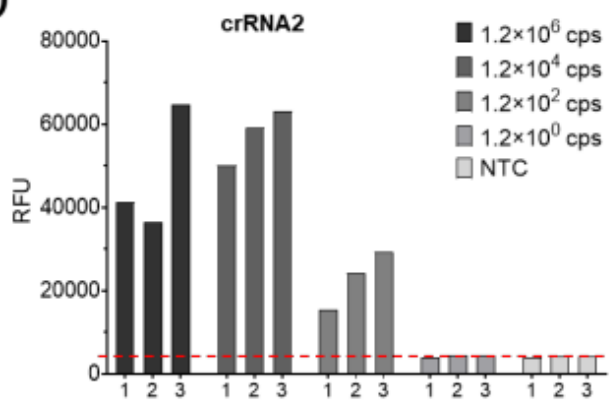

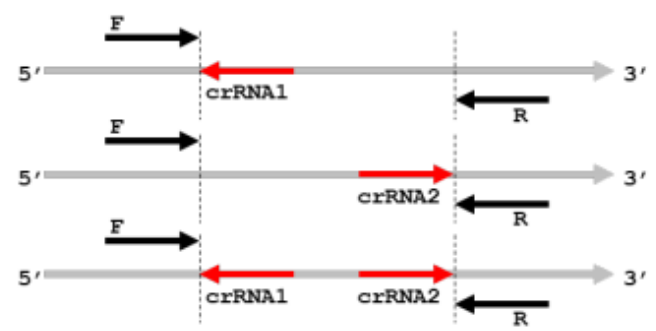

Primers and crRNAs (Product size:137 bp)

F: CAAGCAGCCATGCAAATGTTAAAAGAAACCATC

R: GTAGTTCCTGCTATGTCACTTCCCCTTGGATC

CrRNA1: UAAUUUCUACUAAGUGUAGAUAUCCCAUUCUGCAGCUUCCUCAUU CrRNA2: UAAUUUCUACUAAGUGUAGAUUUGCACCAGGCCAGAUAAGA
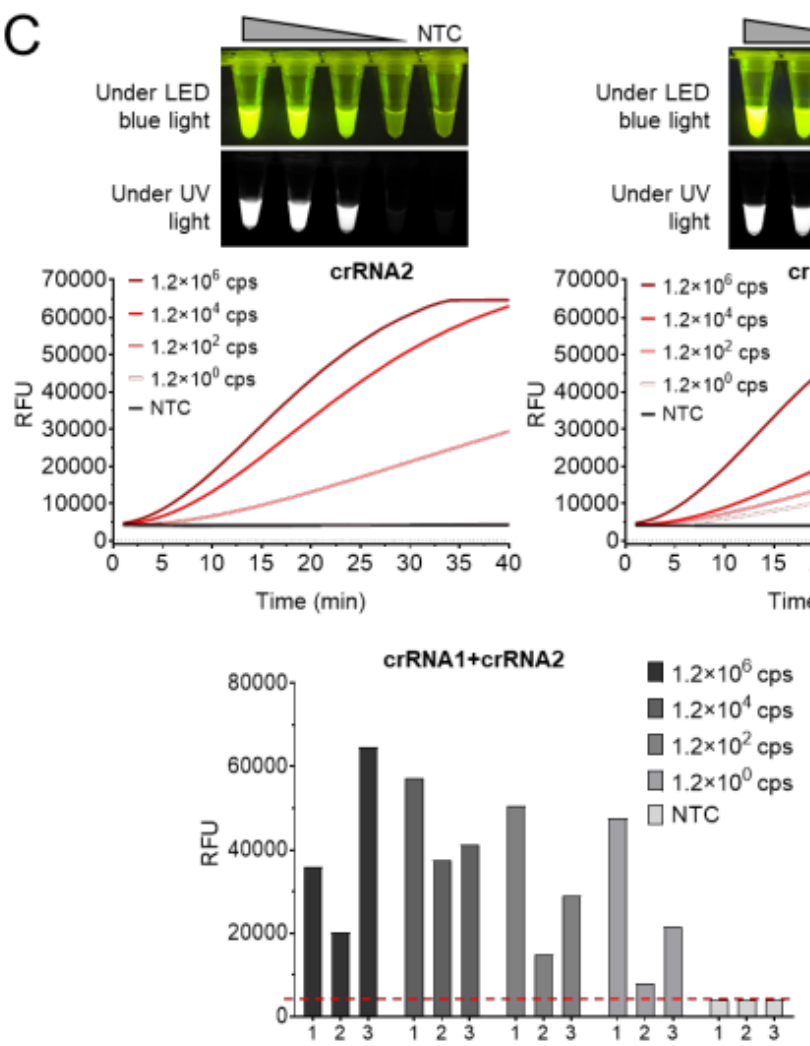

Figure 2

Comparison of the all-in-one CRISPR-Cas12a assay using dual crRNAs or single crRNA. (A) The pUCIDTAMP plasmid containing 300 bp HIV-1 p24 gene cDNA (p24 plasmid) and the sequences of its primers and crRNAs. (B) Real-time fluorescence detection of the all-in-one CRISPR-Cas12a assay using dual crRNAs (crRNA1+crRNA2) or single crRNA (crRNA1/crRNA2). Either $2{ }^{*}$ crRNA1 or $2{ }^{*}$ crRNA2 means doubling its amount. $\mathrm{P}$, the positive control with 1.2× 103 copies of HIV-1 p24 plasmids. Three replicates were run for each reaction with the plasmid. (C) Sensitivities of the all-in-one CRISPR- Cas12a assays with dual crRNAs (crRNA1+crRNA2) or crRNA2 for the detection of various copies of HIV-1 p24 plasmids. (D) Endpoint fluorescence intensity comparisons of the all-in-one CRISPR-Cas12a assays with dual crRNAs (crRNA1+crRNA2) or crRNA2 in three independent experiments. The dashed line indicates the cutoff fluorescence intensity which was the average of the NTC plus two standard deviations. NTC, non- 
template control reaction. Each reaction contained $2 \mu \mathrm{M}$ ssDNA-FQ. Endpoint fluorescence analysis was conducted after 40 min incubation.
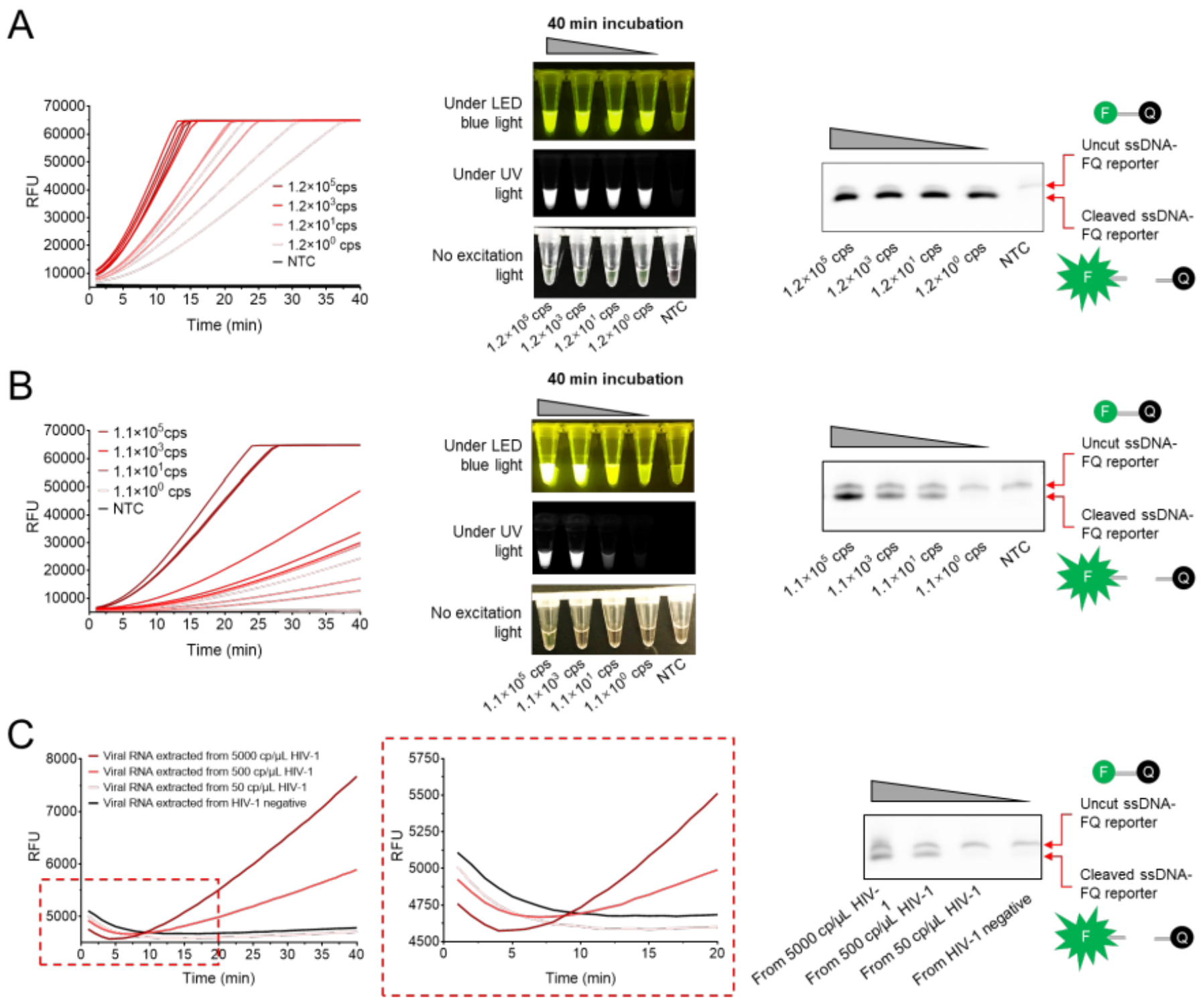

\section{Figure 3}

HIV-1 detection by AIOD-CRISPR/ RT-AIOD-CRISPR assays. (A) Real-time monitoring/endpoint detection of ten-fold serial dilution of HIV-1 p24 plasmid templates and the denaturing PAGE analysis of their ssDNA-FQ products. (B) Real-time monitoring/endpoint detection of ten-fold serial dilution of HIV-1 gag RNA templates and the denaturing PAGE analysis of their ssDNA- FQ products. Three replicates were run for each test. NTC, non-template control reaction. (C) Real- time RT-AIOD-CRISPR assay for the detection of HIV-1 RNA extracted from human plasma samples and the denaturing PAGE analysis of their ssDNAFQ products. 
A

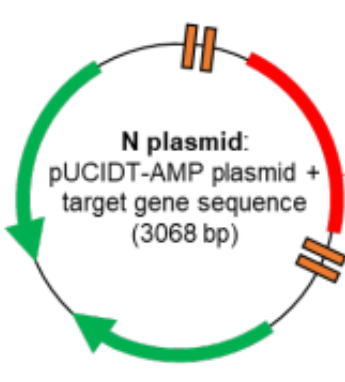

B

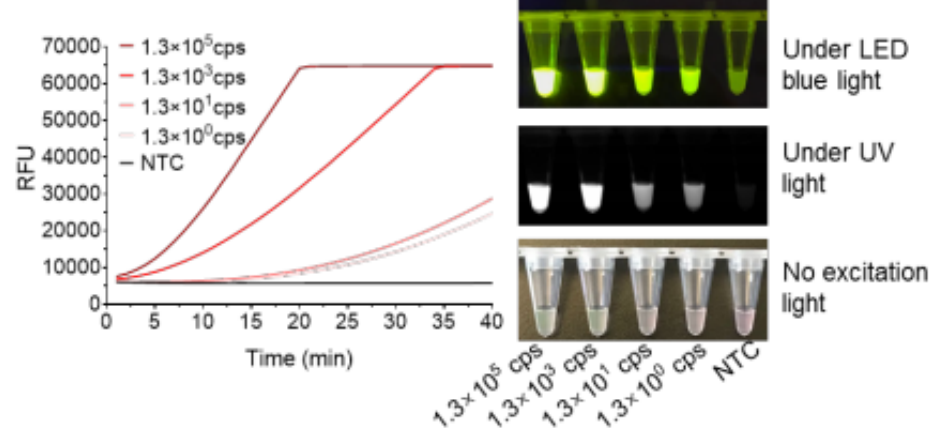

Target: 316 bp SARS-CoV

$2 \mathrm{~N}$ gene cDNA (from 28766

LC528233.1) to 29081 in GenBank No.

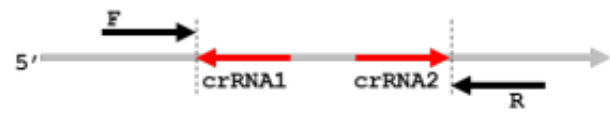

Primers and crRNAs (Product size:121 bp)

F: AGGCAGCAGTAGGGGAACTTCTCCTGCTAGAAT

R: TTGGCCTTTACCAGACATTTTGCTCTCAAGCTG

CrRNA1: UAAUUUCUACUAAGUGUAGAUCAUCACCGCCAUUGCCAGCC CrRNA2: UAAUUUCUACUAAGUGUAGAUUUGCUGCUGCUUGACAGAUU

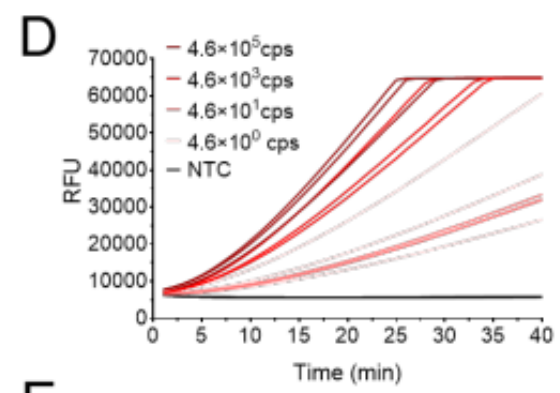

E

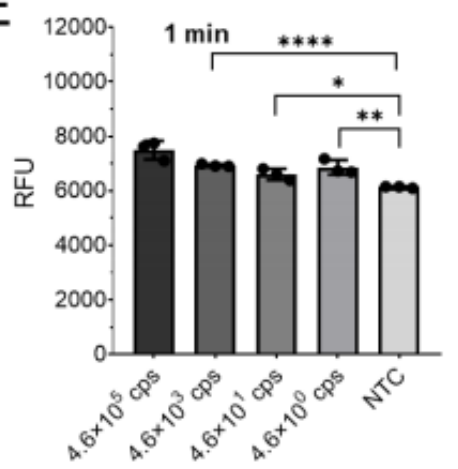

C
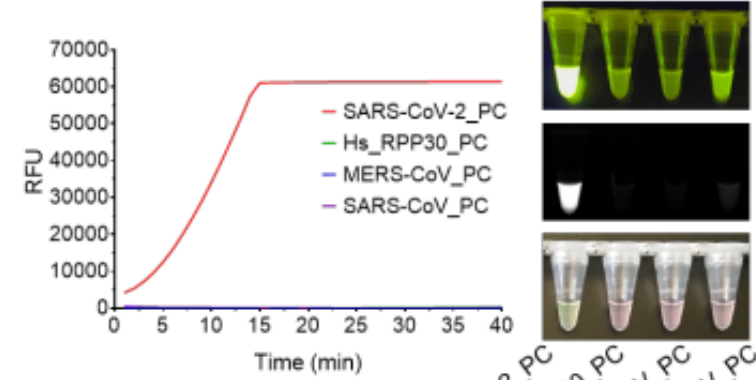
Under LED blue light

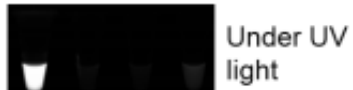
light

No excitation light

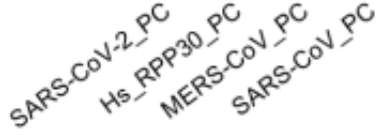

No excitation light

Under UV light

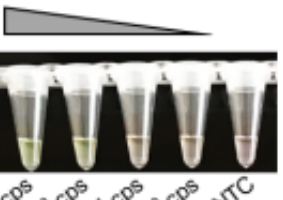

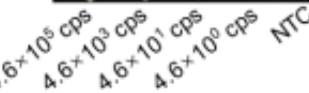

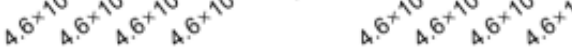

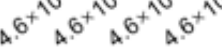
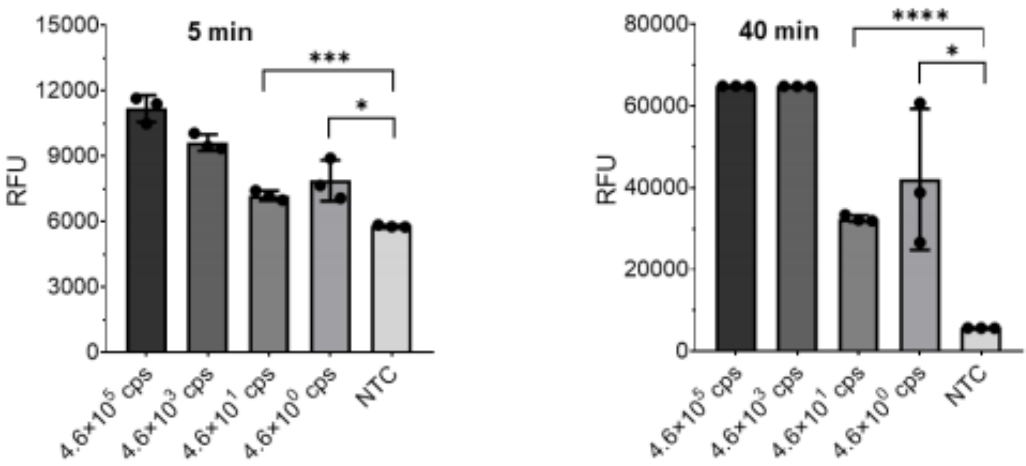

Figure 4

SARS-CoV-2 detection by AIOD-CRISPR assay. (A) The pUCIDT-AMP plasmid containing 316 bp SARSCoV-2 N gene cDNA (N plasmid) and the primers and crRNAs. (B) Real-time AIOD- CRISPR detection with ten-fold serial dilution of SARS-CoV-2 N DNA. (C) Specificity assay of the AIOD- CRISPR assay on SARSCoV-2 N detection. (D) Sensitivity of the AIOD-CRISPR assay in both real- time and endpoint fluorescence/visual detection detections for SARS-CoV-2 N RNA sequences. Three replicates were run for each test. (E) Endpoint fluorescence comparison after 1, 5, and 40 min incubation at $37^{\circ} \mathrm{C}$ for the detection of ten-fold serial dilution of SARS-CoV-2 N RNA templates. Tube images were taken after 40 min incubation. NTC, non-template control reaction. Three replicates were run for each reaction or test. 
Error bars represent the standard deviations at three replicates $(n=3)$. Unpaired two-tailed t-test was used to analyse the difference from NTC. ${ }^{*} P<0.05 ;{ }^{*} \mathrm{P}<0.01 ;{ }^{* \star \star} \mathrm{P}<0.001 ; \star \star \star \star \mathrm{P}<0.0001$. ns, not significant.
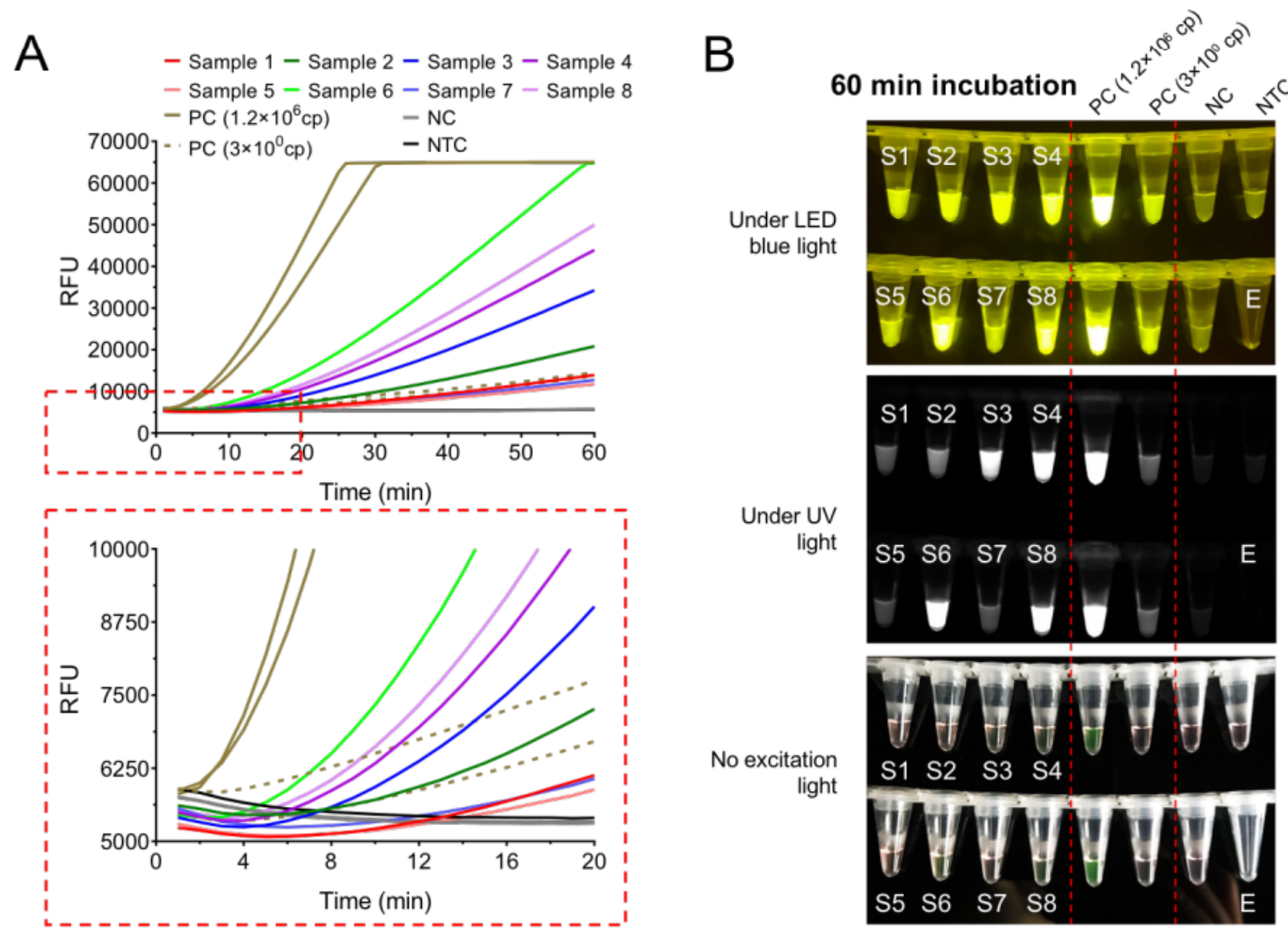

\section{Figure 5}

Detection of SARS-CoV-2 in clinical swab samples by RT-AIOD-CRISPR assay. (A) Real- time RT-AIODCRISPR detection. (B) Endpoint fluorescence/visual detection detections. PC, positive controls with 1.2×106 and 3 copies of SARS-CoV-2 N RNA. S1-S8, clinical sample 1-8. NC, SARS- CoV-2-negative samples. NTC, non-template control reaction. E, Empty tube. 
A

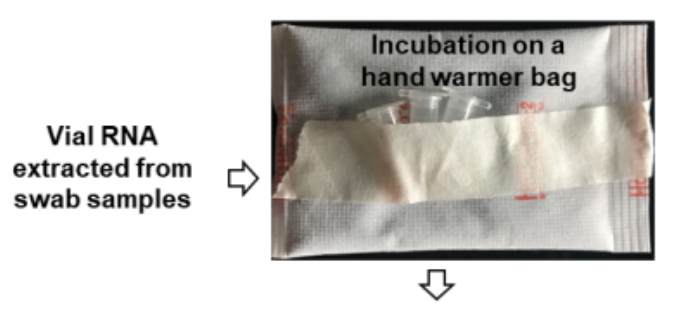

Visual detection in a portable LED blue transilluminator
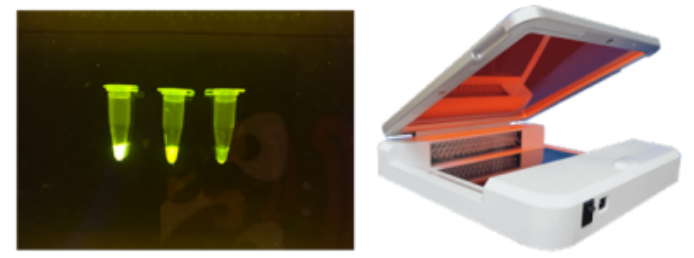

B
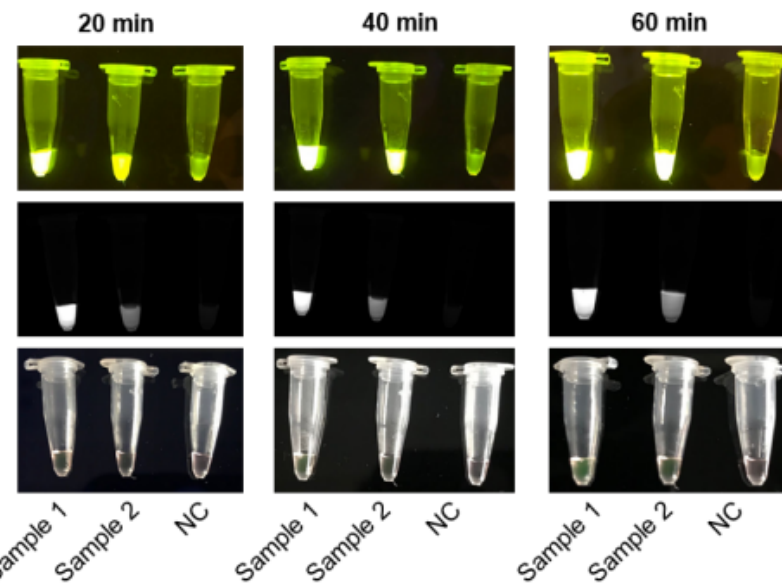

Under LED blue light
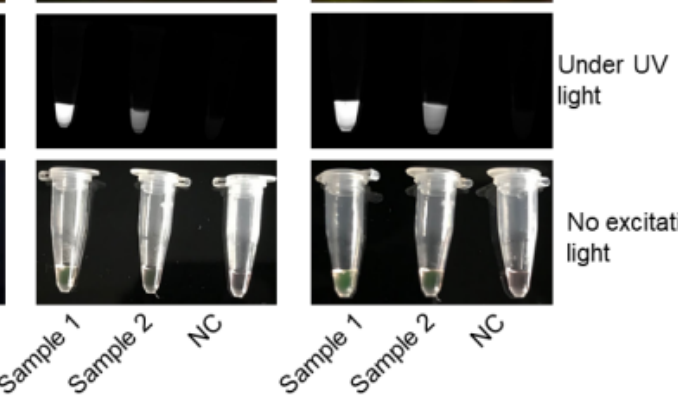

No excitation light

C
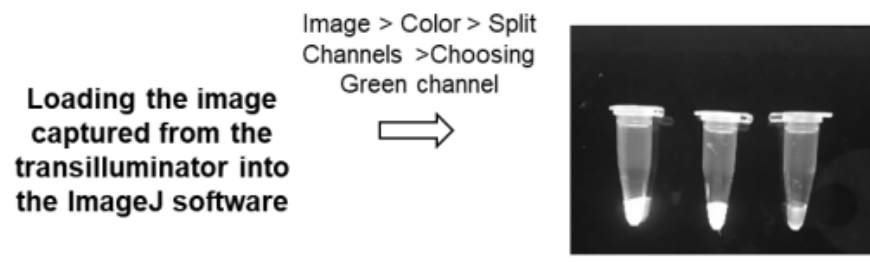

Analysis of the Value of Green

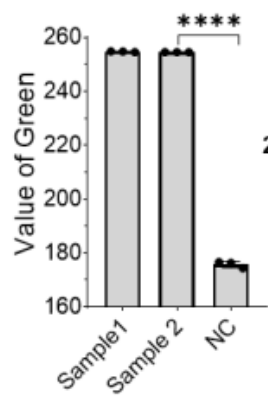

20 min incubation

\section{Figure 6}

Instrument-free COVID-19 diagnostics by RT-AIOD-CRISPR assay. (A) Workflow of the instrument-free POC diagnostics. (B) Visual detection after 20, 40, and 60-min incubation on the hand warmer bag. (C) Analysis of the green value for the fluorescence image (20-min incubation) using the Image J software. NC, the SARS-CoV-2-negative sample. Each measuring was run with three replicates.

\section{Supplementary Files}

This is a list of supplementary files associated with this preprint. Click to download.

- NCOMMS2012493AZSI.pdf 